
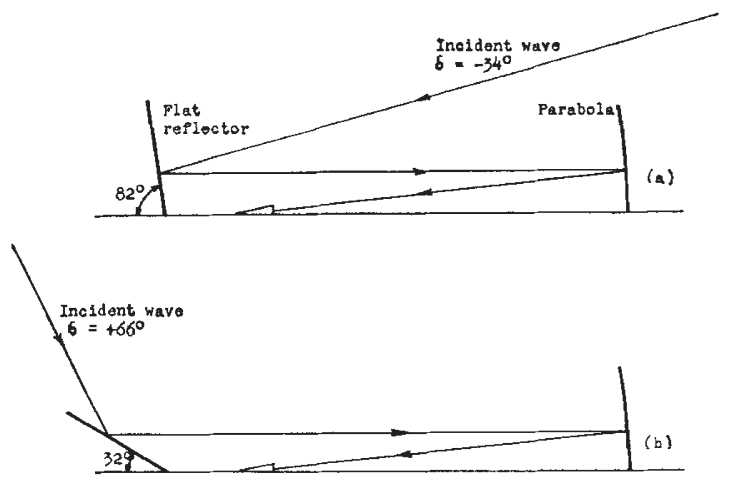

Fig. 5. Diagrams showing extreme positions of the flat reflector and corresponding wave or ray paths

operation of the telescope, but it is hoped that these deficiencies can be remedied at an early date.) This fan beam from the antenna has the same area resolution as a symmetrical pencil beam with a half-power beam-width of about $0 \cdot 2^{\circ}$. However, the fan beam has the advantage over the pencil beam of reducing the time required for a complete scan of the sky by a factor of about $2 \cdot 5$, and also of providing twice the resolution in the direction in which the sky is scanned. A fan beam is also valuable for the reason that it differs in shape from the pencil beam typical of most steerable dish antennas. Thus, comparisons of source and background surveys made with beams of different shape are valuable in detecting spurious effects, since beams of different shape are, in general, subject to different aberrations.

Although no tracking ability is required for most sky-mapping work, a small measure of tracking can be provided in the new telescope by some east-west movement of the feed antenna. Tests of the displacement of the feed antenna on the scale model showed that tracking of sources in hour angle may be possible for at least $10 \mathrm{~min}$. in time at the highest frequency of operation and for more than $1 \mathrm{hr}$. at lower frequencies. With vertically polarized feed antennae the electric field vector is perpendicular to the horizontal ground plane, so that the intensity along the ground plane can be a maximum. Under these conditions the telescope is most efficient and operation is restricted to vertical polarization by providing only vertical wires in the reflecting surfaces of the standing parabola and flat reflector.

The flat reflector is designed to tilt to angles between $32^{\circ}$ and $82^{\circ}$, as illustrated in Fig. 5. A motion of the flat reflector through $50^{\circ}$ (from $32^{\circ}$ to $82^{\circ}$ ) results in a motion of the antenna beam through twice this value, or from a declination of $66^{\circ} \mathrm{N}$. to $34^{\circ} \mathrm{S}$. (total $100^{\circ}$ ). The latitude of the telescope site is about $40^{\circ} \mathrm{N}$. Since the galactic equator is at about $29^{\circ}$ south declination, the telescope will be useful for studies of the region of the galactic nucleus. The fact that the most northerly observable declination is $66^{\circ}\left(24^{\circ}\right.$ from the celestial pole) reduces the sky area coverage only by a small amount, the unobservable area being only about 6 per cent of the observable sky. Since the slant height of the flat reflector is $100 \mathrm{ft}$., while the parabola is $70 \mathrm{ft}$. high, the full aperture of the parabola can be utilized at all declinations south of $40^{\circ} \mathrm{N}$. At the most northern declination of operation $\left(66^{\circ}\right)$, the vertical height of the flat reflector will be 76 per cent of the height of the parabola, reducing the useful aperture of the telescope by 24 per cent under these conditions.

The primary or feed antenna radiation will be tapered in order to reduce the minor lobes of the telescope beam pattern to a minimum. This is important in eliminating spurious responses or ghost sources. An important characteristic of the design is that signal pick-up from the ground is minimized, the telescope antenna beam pattern being always directed skyward with the parabolic and flat reflectors providing a measure of shielding from interfering terrestrial signal sources.

The fact that the main part of the telescope has only one moving part, the flat reflector, the position of which can be accurately controlled, means that it will be possible to measure positions of celestial sources to a high precision and with good repeatability.

\section{Conclusion}

The new Ohio State University telescope will be an experimental working model of the standing parabola tiltable flat reflector design which should provide useful information for the construction of similar telescopes of even larger size. It is hoped that the telescope will be completed during 1960, at which time a programme of sky-mapping will be initiated that will cover a wide range of frequencies. More detailed sky maps and more accurate spectra of radio sources will be objectives of the surveys. Instrumentation for hydrogen line observations is also to be installed, and observations conducted of the distribution of neutral hydrogen in our galaxy.

1 Kraus, J. D., "Antennas", 485 (McGraw-Hill Book Co., Inc., New York and London, 1950'.

2 Nash, R. T., master's thesis, Ohio State University (1955).

${ }^{3}$ Kraus, J. D., and Nash, R. T., Ohio State University Radio Observaraus, J. D., and Nash, R. T

${ }^{4}$ Kraus, J. D., Ko, H. C., Nash, R. T., and Stoutenberg, D. V., Astro. J., Bi, 182 (1956).

\title{
RADIOCARBON DATING
}

\footnotetext{
CONTRIBUTIONS were made by representatives 1 of each of the three laboratories in England which are actively engaged in radioactive dating to a joint session of Sections $A$ (Physics) and $H$ (Anthropology and Archæology) of the British Association at the recent meeting at York. They combined to provide a picture of the basis of the method and its limitations, the trend of future development in instrumentation, and the capabilities of the method as applied to individual problems in the late Quat. ernary history of the British Isles.
}

In reviewing the basis of the method of radiocarbon dating, Mr. H. Barker, of the British Museum Research Laboratory, was concerned with the difficulties which had sometimes arisen as a result of the lack of common ground between the archæologist and the physicist. Lack of familiarity with statistical concepts and misunderstandings as to the nature of radioactive decay had led to many misconeeptions and the validity of the method had been questioned on wrong premises. The method depends on the fact, predicted and verified by Libby, that the radio- 
active carbon isotope of mass 14 (known as radiocarbon or carbon-14) is being continually created in the upper atmosphere as a result of the action of cosmic rays. Consequently it is distributed throughout the world-wide carbon-exchange reservoir which comprises the atmosphere, the oceans, and all plant and animal life. Libby produced sound theoretical argument, backed by practical measurements, for assuming that the carbon-14 is evenly distributed throughout the exchange reservoir and that the level of radioactivity due to this cause has remained the same for many thousands of years. This assumption is fundamental to radiocarbon dating; when an organism dies it is cut off from the exchange reservoir, the possibility of it replenishing its carbon-14 by vital processes ceases, and its store of carbon-14 diminishes at a rate determined only by the half-life (about 5,600 years). One can therefore calculate the time which has elapsed since it was alive, from a measurement of its residual carbon-14 activity, provided that one can assume that its original activity was the same as that of a reference sample which has been recently removed from the reservoir. The measurement is exceedingly difficult, and limitations in accuracy, imposed by statistical fluctuations in the measurement of radioactivity, result in an error term being associated with each radiocarbon date. The correct interpretation of the significance of this error-term requires some familiarity with statistical concepts, and lack of appreciation of this has led to misunderstandings.

In the ten years which have elapsed since Libby announced his first successes, there has been a revolution in instrumentation. Nowadays, the measurement of radioactivity can be made to a much higher accuracy, and the question arises as to whether the basic assumption of the method still holds good at this new level of accuracy. In fact, it seems that a number of minor effects can combine to limit the accuracy. Craig showed that isotopic fractionation in plants can result in fluctuations of up to \pm 1 per cent in carbon-14 activity from the mean value. This corresponds to an error of \pm 80 years. For tunately, it is possible to apply corrections for this effect based on mass-spectrometric measurements of the ratios of the other carbon isotopes in the sample and the standard. Systematic errors, mainly due to differences in 'recent' standards, have caused difficulties in comparing results between different laboratories. In most cases, the errors are quite small, but steps are now being taken to eliminate these differences. Very recently, de Vries has shown that the level of activity in the exchange reservoir has suffered cyclic changes of about \pm 1 per cent during the past 500 years. So far as one can see at present, this effect definitely limits the aceuracy obtainable in dating to about 100 years.

Apart from these theoretical limitations, it must not be forgotten that the choice of sample plays an important part in the real accuracy obtainable. The archæologist has to relate the death of an organism (which is what is actually measured) to an event in which he is interested. The environment of the sample is not unimportant, the possibility of sample contamination must not be overlooked, and suitable laboratory treatment applied when necessary to remove contaminating materials such as rootlets and humic acids.

Mr. W. J. Callow, of the National Physical Laboratory, also referred to the factors which limit the accuracy of the method, but pointed out that, despite these limitations, the radiocarbon method remains a very valuable technique for chronological studies in archæology, geology, botany, oceanography and allied fields. There are now nearly forty laboratories distributed throughout the world which have been, or are, currently engaged in this form of dating. Some time ago, it was realized that there was a need for a dating service to be made generally available to workers in Great Britain. The laboratories at the Botany School, Cambridge, and the British Museum were occupied in the main with their own programmes, and it was considered appropriate that the new service should be set up at the National Physical Laboratory.

The instrumental difficulties are considerable in that the measurement is concerned with levels of radiocarbon concentration ranging from one part in a million million down to zero. In the case of recent carbon, this corresponds to the breakdown of about fifteen carbon-14 atoms per min. per gm. of carbon. Detecting and recording these disintegrations requires special techniques due to the low energy of the betapartieles and the low counting-rates involved.

In all methods, the first process involved consists of converting the sample to carbon dioxide, either by combustion, in the case of organic material, or by acid treatment, in the case of carbonates. In Libby's original method the carbon dioxide was reduced to solid carbon which had to be placed inside a counter because of the low beta-particle energy. Due to the low efficiency of the counter under these conditions and the possibility of contamination with radioactive fall-out and naturally occurring radon, this technique has now been abandoned.

Both de Vries in Holland and Fergusson in New Zealand have shown that carbon dioxide itself, if sufficiently pure, can be used as the operating gas in a proportional counter, and this technique is being adopted at the National Physical Laboratory. As little as one part per million of electronegative impurity such as oxygen or oxides of nitrogen in the carbon dioxide can prejudice the reliable operation of the counter, and hence the accuracy of the measurement. The gas preparation equipment at the National Physical Laboratory is therefore designed to produce carbon dioxide of an extremely high degree of purity.

Carbon dioxide is not the only possible counter filling; methane and acetylene are in use in some laboratories. Both involve rather more preparatory work, but they are more tolerant of the presence of impurities. Acetylene has the advantage of having two atoms of carbon in its molecule, and therefore, under a given set of conditions, the use of it enables one to place twice as much carbon in the counter compared with carbon dioxide or methane methods.

Liquid-scintillation counting also provides a possible approach to the problem of measurement, but difficulties in chemical preparation prevent its general adoption.

The actual measurements are complicated by the fact that, in normal circumstances, the background counting-rate of the counter would be very much greater than that due to the sample itself, and steps must be taken to reduce its value. A heavy steel sereen with walls 8 in. thick is used to eliminate the effect of local gamma-radiation. The screen at the National Physical Laboratory, which is large enough to accommodate a number of counters, weighs more than 23 tons. The penetrating meson component of 
cosmic radiation, against which the steel screen has no effect, is cancelled out by a ring of Geiger counters surrounding the sample counter and connected in anti-coincidence. Other screening precautions which have been shown to be advisable and which are incorporated in the equipment at the Laboratory include a mercury shield, which reduces the background due to traces of radioactivity in the steel screen, and a layer of paraffin wax mixed with boric acid which reduces the component due to neutrons which are in turn induced in the screen by the action of the corpuscular component of cosmic radiation. This neutron flux will also be monitored continuously. Since accurate measurement of these very low dis. integration-rates necessarily takes a long time, great emphasis has been placed on the reliable operation of the associated electronic equipment. The instrumentation at the National Physical Laboratory is based on the use, wherever possible, of high-stability circuits as used in computers, and of transistors instead of thermionic valves. The equipment includes a comprehensive system for automatic recording, and a mass spectrometer has also been purchased in order that corrections can be applied for isotopic fractionation effects. The equipment is nearing completion and should be in operation in the near future.

Dr. H. Godwin described how the method is being used in the Cambridge Sub-department of Quaternary Research to give an absolute time-scale to the history of events during the Late Quaternary period, that is, the past 35,000 years or so. The apparatus, designed and built by Dr. E. H. Willis, consists of a proportional gas counter of about 2-litres volume. It is shielded by 7 tons of zinc and lead and an anticoincidence array of Geiger tubes. It has a net contemporary count-rate of 28 counts per min. and a steady background of $20 \cdot 7$. The equipment was acquired as a result of the generosity of the Nuffield Foundation, but is now maintained by the University of Cambridge.

The oldest sample dated so far was from the Arctic Plant Bed from the Lea Valley. This gives a date of 26,000 B.c. for the time when the mammoth was still alive in Britain. A date from a later horizon in the valley of the Colne, a tributary of the Lea, shows that the full Glacial Period must have persisted until $c .11,500$ B.c. After the glaciation, there came a period of climatic oscillation, the so-called Allerød Period. The effects of this change can be recognized in deposits at numerous sites throughout the British Isles, Carbon dating on material from such sites shows that there was a temporary mild period between 10,000 and 8,800 B.C., followed by a return of cold for about 500 years. These British dates are in excellent agreement with dates from north-west Europe. As a result, British events are now closely tied up with events at the end of the ice age in Europe.

Following the Allerød oscillation came the climatic improvement of the Post Glacial Period. In the past, pollen analysis has provided a relative time-scale for this period since the characteristic changes in forest composition have permitted the establishment of a sequence of pollen zones applicable, with care, to the whole of the British Isles. A series of twelve datings from a site in Cumberland gives a very consistent series of dates for the successive pollen zones. It remains to be seen how far these pollen boundaries are truly synchronous across the British Isles or Western Europe.

The increased temperature at the end of the last glaciation caused melting of the ice sheets and a rapid rise in ocean-level. One effect of this was the flooding of the North Sea and the isolation of Britain from the European mainland. Dates measured in Cambridge on submerged peat-beds from around the coast of Britain, together with those made at other laboratories, show that a rapid eustatic rise of sea-level was in progress between 12,000 and 4,000 B.c. and that the level rose by more than $200 \mathrm{ft}$.

In the derelict raised bogs of Somerset, several prehistoric wooden trackways have been recorded and excavated. They appear to have been built in the late Bronze Age at a time when increasing wetness of climate induced flooding of bogs and valleys and caused Bronze Age man to construct these wooden causeways. Radiocarbon dating of seven such structures in Somerset and three others in Lancashire, Cambridgeshire and Lincolnshire show that they were built in the period 500-900 B.c.

The picture which emerged from this discussion is a very hopeful one for the future of radiocarbon dating in Britain. Although the method has certain limitations, the work described by Dr. Godwin illustrates its great value as a tool for establishing an absolute chronological framework to prehistoric events, and the news that Britain's present limited capacity for radiocarbon dating is to be augmented by the considerable resources of the National Physical Laboratory will be welcomed in many quarters. The dates mentioned in the discussion appear in a paper in the American Journal of Science Radiocarbon Supplement, Vol. 1 (1959), the new international organ for the publication of radiocarbon dates.

Harold Barker

\section{MECHANISM OF HORMONE ACTION}

\section{By PROF. AARON B. LERNER}

Section of Dermatology, Department of Medicine, Yale University School of Medicine, New Haven, Conn.

CELLS have specific functions, for example,
adrenal cortical cells produce hydrocortisone,
nerve cells form noradrenaline, melanocytes make
melanin. During their early development cells have
in common the capacity to produce substances such
as glycogen and protein. The formation of all these
end-products depends upon the reaction of specific
enzymes with their substrates. Such enzymically catalysed syntheses usually occur in soluble extracts and homogenates which do not contain intact cells. The formation of cellular end-products also is controlled by hormones that require a high degree of cellular organization in order to function. Although the synthesis of a cell's products is regulated by both enzymic and hormonal reactions, these reactions are not related directly to one another. The hormones 